\title{
Acute Kidney Injury Due to Diarrheal Illness Requiring Hospitalization: Data from the National Inpatient Sample
}

\author{
Christina Bradshaw, MD' ${ }^{7}$, Yuanchao Zheng, $\mathrm{MS}^{7}$, Samuel A. Silver, MD, MSc ${ }^{2}$, \\ Glenn M. Chertow, MD, MPH ${ }^{7,3}$, Jin Long, $P h D^{7}$, and Shuchi Anand, MD, $\mathrm{MS}^{\mathrm{T}}$
}

'Division of Nephrology, Department of Medicine, Stanford University School of Medicine, Stanford, CA, USA; ${ }^{2}$ Kingston Health Sciences Center, Division of Nephrology, Queen's University, Kingston, ON, Canada; ${ }^{3}$ Department of Health Research and Policy, Stanford University School of Medicine, Stanford, CA, USA.

BACKGROUND: Diarrheal illness is a major reason for hospitalization, but data on consequent acute kidney injury (AKI) are sparse.

OBJECTIVE: To determine the incidence of AKI in infectious and non-infectious diarrheal illness requiring hospitalization and to identify correlates and outcomes of diarrhea-associated AKI.

DESIGN: Using data from the 2012 National Inpatient Sample (NIS), we created a cohort of patients with a primary diagnosis of diarrheal illness. Diarrheal illness, disease correlates, and AKI were defined by ICD-9 diagnosis codes. We used logistic regression with backward variable selection to determine factors independently associated with AKI in infectious and non-infectious diarrheal illness, as well as to determine the association of AKI with in-hospital mortality. We used generalized linear models to assess differences in length of stay and costs of hospitalization.

MAIN MEASURES: The primary outcome was AKI in hospitalized diarrheal illness. Secondary outcomes were inhospital mortality, length of stay, and cost of hospitalization associated with AKI.

KEY RESULTS: One in ten adults hospitalized with diarrheal illness experienced AKI, with higher incidence rates in older adults. Chronic kidney disease (CKD) and hypertension were associated with increased odds of AKI (all diarrhea OR 4.81, 95\% CI 4.52 to 5.12 and OR 1.33, 95\% CI 1.27 to 1.40 , respectively). AKI in diarrheal illness was associated with substantial increase in mortality (OR $5.05,95 \%$ CI 4.47 to 5.72), length of stay (mean increase 1.7 days [95\% CI 1.6 to 1.8]), and cost of hospitalization (mean increase \$4411 [95\% CI 4023 to 4800]).

CONCLUSION: Acute kidney injury is common and consequential among patients hospitalized for diarrheal illness. Persons with $\mathrm{CKD}$ and hypertension are the most susceptible, possibly due to diminished renal reserve and exacerbating effects of treatment with diuretics and reninangiotensin-aldosterone system blockers. Proactive management of these unique pharmacologic and physiologic factors is necessary to prevent AKI in this vulnerable population.

Electronic supplementary material The online version of this article (https://doi.org/10.1007/s11606-018-4531-6) contains supplementary material, which is available to authorized users.

Received November 15, 2017

Revised March 20, 2018

Accepted May 29, 2018

Published online June 18, 2018
KEY WORDS: renal disease; clinical epidemiology; hospital medicine; prevention.

J Gen Intern Med 33(9):1520-7

DOI: $10.1007 / \mathrm{s} 11606-018-4531-6$

(C) Society of General Internal Medicine 2018

\section{INTRODUCTION}

Diarrhea is one of the most common illnesses requiring hospitalization globally. ${ }^{1,2}$ In the USA, hospitalizations related to Clostridium difficile have nearly tripled between 2001 and $2011{ }^{3}$, with persons above the age of 65 years especially vulnerable. ${ }^{4}$ Hospitalizations due to ischemic colitis have quadrupled over the past three decades, ${ }^{5}$ and rates of diverticulitis are rising by $5 \%$ per year among young adults. ${ }^{6,7}$

In view of the scope and severity of diarrheal disease, surprisingly little is known about the incidence and correlates of associated acute kidney injury (AKI), one of its most serious consequences. The volume depletion that accompanies severe diarrheal illness is a well-known risk factor for AKI, but other factors, including multimorbidity and polypharmacy, are also likely culprits. Further characterizing the burden of AKI in diarrheal illness is important because AKI has been associated with mortality, prolonged lengths of hospital stay, higher costs, and the risk of developing chronic kidney disease (CKD) ${ }^{8-10}$

We used data from the National Inpatient Sample (NIS) to determine the incidence of AKI associated with hospitalized diarrheal illness in the USA. We present estimates for incidence of AKI by age-group ( $<45,45$ to 64,65 to 79 , and 80 and above), and by etiology (non-infectious versus infectious), and report correlates and consequences (mortality, length of stay and cost) of AKI accompanying hospitalized diarrheal illness.

\section{METHODS}

\section{Study Population}

We extracted data from the NIS, a nationally representative administrative database of hospitalizations in the USA created by the Agency for Healthcare Research and Quality as part of the Healthcare Cost and Utilization Project (HCUP). ${ }^{11}$ The 
NIS is the largest all-payer inpatient-care database, and contains a $20 \%$ stratified sample of yearly discharge data from short-term, non-federal, non-rehabilitation hospitals. Data are stratified according to geographic region, location (urban/rural), teaching status, ownership, and number of hospital beds. Each hospitalization is treated as an individual entry in the database, such that individual patients who are hospitalized multiple times may be present in the NIS multiple times. The NIS includes demographic variables, diagnoses, procedures, length of stay, and hospital charges. Sample weights are provided to allow for the generation of national estimates, along with information necessary to calculate the variance of estimates. Hospital-specific charge-to-cost ratios are available to calculate costs. We used the 2012 NIS subset, the most recent year available at the time of data analysis. The 2012 NIS subset contained administrative data from over seven million hospitalizations, representing more than 4000 hospitals, 44 states, and $95 \%$ of the US population.

\section{Primary Exposure-Infectious and Non- infectious Diarrhea}

We created a cohort of all hospitalized adults with a primary diagnosis of diarrheal illness (infectious and non-infectious). We evaluated infectious and non-infectious diarrheal illness as separate clinical entities, with the hypothesis that risk factors for development of AKI in patients with a diagnosis of infectious verus non-infectious diarrhea may differ substantially.

For infectious diarrhea, we used the following International Classification of Disease, 9th revision, clinical modification (ICD-9 CM) codes from 2012: 001, 002, 003.0, 004, 005, 007, 008, 009, 084.6, 112.85, and 487.8. For non-infectious diarrhea, ICD-9 CM codes included 306.4, 536.8, 555, 556, 557, 558, 562.01, 562.03, 562.11, 562.13, 564.1, 564.2, 564.4, $564.5,564.81,564.9$, and 579 . We excluded the code for "diarrhea NOS" (787.91) since it was rarely used. We crosschecked the diarrhea codes with existing literature. ${ }^{12-16} \mathrm{We}$ excluded patients with end-stage renal disease (ESRD), using an approach we and others have outlined previously ${ }^{17-19}$ and which has been shown to produce high sensitivity and specificity, as well as high positive and negative predictive values (all $\geq 90 \%$ ) for differentiating dialysis-requiring AKI (AKI-D) from maintenance dialysis. ${ }^{19}$

\section{Primary Outcome-Acute Kidney Injury}

We identified episodes of AKI using the ICD-9 diagnosis code 584.x. This administrative code for AKI has low sensitivity, but specificity of approximately $99 \%$, suggesting that our cohort should include few false positives, and represents a more severe spectrum of AKI compared to serum creatinine criteria. ${ }^{19,20}$ For example, the median (25th, 75 th percentile) change in serum creatinine from baseline is estimated at 1.2 (0.7 to 2.1$) \mathrm{mg} / \mathrm{dL}$ compared with 0.2 (0.1 to 0.2$) \mathrm{mg} / \mathrm{dL}$ for patients without an administrative code for AKI. ${ }^{19}$

\section{Secondary Outcomes}

To describe the consequences of AKI in hospitalized diarrheal illness, we compared in-hospital mortality, length of stay, and costs for groups with and without AKI. We also tested for effect modification by age and diarrhea type.

\section{Covariates}

We selected comorbidities of interest based on 2012 ICD-9 diagnosis codes, relying on existing literature and grouping by organ system. ${ }^{21-24}$ Any ICD-9 code that reflected an acute condition was excluded. Supplementary Table 1 provides the ICD-9 codes used to create comorbidity definitions as well as grouping of comorbidities into organ system. Hospital-level variables included geographic region, bed number, and teaching status using pre-determined NIS definitions. ${ }^{11}$

\section{Statistical Analysis}

We summarized baseline characteristics of the study participants using descriptive statistics, stratifying by diarrhea type (infectious versus non-infectious). Continuous variables were expressed as mean (standard deviation [SD]) or if not normally distributed, median (10th, 90th percentile) where appropriate. Categorical variables were expressed as proportions. When examining correlates of AKI associated with diarrheal illness, we considered an array of demographic, clinical, and hospital characteristics as presented in Table 1. We used logistic regression with backward variable selection (retention $p$ value criterion $<0.10$ ) to determine correlates associated with AKI. We ran separate models by diarrhea type (infectious versus non-infectious). We calculated odds ratios (OR) and 95\% confidence intervals $(95 \% \mathrm{CI})$ of the associated correlates and ranked them by their absolute standardized-beta-coefficient to determine their relative contribution to the risk of developing our primary outcome: AKI in hospitalized diarrheal illness. To determine the consequences of AKI in this cohort, we conducted secondary analyses of in-hospital mortality, length of hospital stay, and cost, also using backward selection methods. We calculated ORs and 95\% CIs to show the association between AKI versus no AKI in infectious, non-infectious and all diarrheal illness and mortality rates. Due to the skewness of length of stay and cost data, we used a generalized linear model (GLM) with a gamma distribution and a log link to obtain the adjusted mean increase in length of stay and cost for each diarrhea subgroup and all diarrhea, adjusting for demographic, clinical, and hospital characteristics. ${ }^{25,26}$

All analyses presented account for the NIS survey design (weighting and stratification) and subpopulation measurements to generate national estimates. We created the cohort and conducted the analyses using the Statistical Analysis System (SAS) software, version 9.4 (SAS Institute, Cary, NC) and StataMP, version 14.0 (Stata Corporation, College Station, TX). 
Table 1 Demographics, Chronic Conditions and Hospital Characteristics of Adults Admitted with Diarrheal Illness

\begin{tabular}{|c|c|c|c|}
\hline & $\begin{array}{l}\text { Infectious diarrhea } \\
N=\mathbf{2 1 4 , 0 3 0} \text { weighted } \%\end{array}$ & $\begin{array}{l}\text { Non-infectious diarrhea } \\
N=\mathbf{5 2 5 , 3 5 5} \text { weighted } \%\end{array}$ & $\begin{array}{l}\text { All diarrhea } \\
N=739,385 \text { weighted } \%\end{array}$ \\
\hline \multicolumn{4}{|l|}{ Demographics } \\
\hline Age, years $($ mean $\pm \mathrm{SD})$ & $63.6 \pm 0.1$ & $57.8 \pm 0.1$ & $59.5 \pm 0.1$ \\
\hline $18-44$ & 18.4 & 25.0 & 23.1 \\
\hline $45-64$ & 28.0 & 36.4 & 34.0 \\
\hline $65-79$ & 27.7 & 24.3 & 25.3 \\
\hline $80+$ & 25.9 & 14.3 & 17.6 \\
\hline \multicolumn{4}{|l|}{ Sex } \\
\hline Female & 64.9 & 61.6 & 62.6 \\
\hline \multicolumn{4}{|l|}{ Race } \\
\hline White & 72.0 & 71.7 & 71.8 \\
\hline Black & 10.9 & 10.1 & 10.4 \\
\hline Hispanic & 7.8 & 9.1 & 8.7 \\
\hline Asian/Pacific Islander & 1.4 & 1.3 & 1.3 \\
\hline Native American & 0.6 & 0.5 & 0.5 \\
\hline Other & 2.5 & 2.6 & 2.6 \\
\hline \multicolumn{4}{|l|}{ Household income } \\
\hline Less than 39,000 & 26.9 & 27.4 & 27.2 \\
\hline $39,000-47,999$ & 23.6 & 24.3 & 24.1 \\
\hline $48,000-62,999$ & 24.3 & 23.9 & 24.0 \\
\hline Above 63,000 & 23.2 & 22.5 & 22.7 \\
\hline \multicolumn{4}{|l|}{ Hospital details } \\
\hline \multicolumn{4}{|l|}{ Hospital ownership } \\
\hline Government, non-federal & 10.3 & 10.8 & 10.7 \\
\hline Private, non-profit & 76.6 & 74.1 & 74.9 \\
\hline Private, investor-owned & 13.0 & 15.1 & 14.5 \\
\hline \multicolumn{4}{|l|}{ Expected primary payer } \\
\hline Medicare & 59.4 & 42.9 & 47.7 \\
\hline Medicaid & 9.3 & 9.7 & 9.6 \\
\hline Private, $\mathrm{HMO}$ & 23.9 & 36.5 & 32.8 \\
\hline Self-pay & 4.7 & 6.9 & 6.3 \\
\hline No charge & 0.4 & 0.7 & 0.6 \\
\hline Other & 2.3 & 3.0 & 2.8 \\
\hline \multicolumn{4}{|l|}{ Region } \\
\hline Northeast & 23.2 & 21.3 & 21.9 \\
\hline Midwest & 23.0 & 22.9 & 23.0 \\
\hline South & 36.7 & 39.3 & 38.6 \\
\hline West & 17.1 & 16.5 & 16.6 \\
\hline \multicolumn{4}{|l|}{ Location/teaching status } \\
\hline Rural & 12.8 & 14.2 & 13.8 \\
\hline Urban, non-teaching & 41.7 & 42.4 & 42.2 \\
\hline Urban, teaching & 45.5 & 43.4 & 44.0 \\
\hline \multicolumn{4}{|l|}{ Bed size } \\
\hline Small & 15.9 & 16.2 & 16.1 \\
\hline Medium & 26.8 & 27.9 & 27.6 \\
\hline Large & 57.3 & 55.9 & 56.3 \\
\hline \multicolumn{4}{|l|}{ Chronic conditions } \\
\hline CKD & 18.0 & 8.2 & 11.1 \\
\hline HTN & 43.5 & 42.9 & 43.1 \\
\hline Other renal/GU & 6.4 & 3.9 & 4.6 \\
\hline Cardiovascular & 38.8 & 25.5 & 29.4 \\
\hline Weight & & & \\
\hline Overweight & 8.3 & 10.5 & 9.8 \\
\hline Underweight & 5.1 & 3.1 & 3.6 \\
\hline Pulmonary & 21.7 & 17.2 & 18.5 \\
\hline Diabetes & 26.0 & 18.5 & 20.7 \\
\hline Other endocrine & 2.1 & 1.4 & 1.6 \\
\hline IBD & 3.6 & 18.4 & 14.1 \\
\hline Other gastrointestinal & 9.5 & 9.2 & 9.3 \\
\hline Neurologic/neuromuscular & 14.4 & 6.1 & 8.5 \\
\hline HIV/AIDS & 1.1 & 0.4 & 0.6 \\
\hline Rheumatologic/immunologic & 10.0 & 6.9 & 7.8 \\
\hline Hematologic, BMT & 29.4 & 20.1 & 22.8 \\
\hline Solid organ transplant & 1.6 & 0.5 & 0.8 \\
\hline Solid tumors & 6.7 & 3.9 & 4.7 \\
\hline Admission details & & & \\
\hline Elective & 4.8 & 13.9 & 11.3 \\
\hline Non-elective & 94.9 & 85.8 & 88.4 \\
\hline Missing & 0.3 & 0.3 & 0.3 \\
\hline Hospitalization outcomes & & & \\
\hline Cost of stay, dollars $\dagger$ & $5760(2703-14,577)$ & $6090(2865-18,539)$ & $5983(2818-17,395)$ \\
\hline Length of stay, days ${ }^{\dagger}$ & $3.0(0.8-8.7)$ & $2.9(0.8-8.2)$ & $2.9(0.8-8.3)$ \\
\hline Mortality & 1.3 & 1.1 & 1.2 \\
\hline
\end{tabular}

$H M O$, health maintenance organization; CKD, chronic kidney disease; HTN, hypertension; GU, genitourinary; IBD, inflammatory bowel disease; BMT, bone marrow transplant

${ }^{\dagger}$ Expressed as median, 10th to 90th percentile 


\section{RESULTS}

\section{Incidence of AKI in Diarrheal Illness}

Table 1 shows demographic, clinical, and hospital characteristics of adults admitted with a primary diagnosis of diarrhea in 2012. The most common ICD-9 diagnosis code associated with infectious diarrhea was 008 (comprising $82.0 \%$ of the infectious diarrhea cohort), which includes diarrhea caused by both bacterial (e.g., Clostridium difficile) and viral (e.g. rotavirus) infections. For non-infectious diarrhea, the most common diagnosis codes were for diverticulitis (ICD-9 code 562.11, comprising 42.1\%) and vascular insufficiency of the intestine (ICD-9 code 557, comprising 11.4\%). Patients admitted with infectious diarrhea were nearly 10 years older on average than patients admitted with non-infectious diarrhea. Nearly twothirds $(62.6 \%)$ of hospitalizations for diarrheal illness were in women. Persons admitted with a primary diagnosis of infectious diarrhea were more likely to be coded as having CKD, immunodeficiency (HIV/AIDS, transplant, malignancy) and neurologic diseases relative to persons with non-infectious diarrhea. Persons admitted with non-infectious diarrhea were more likely to have a secondary diagnosis of inflammatory bowel disease (IBD) than those admitted for infectious diarrhea. A significantly higher proportion of persons with a primary diagnosis of non-infectious diarrhea were admitted in an elective capacity (13.9 versus $4.8 \%$ ). Volume depletion was coded more commonly in persons with infectious diarrhea (35.6\% in infectious versus $14.3 \%$ in non-infectious diarrhea). Prevalence of sepsis and shock was similar (3.4\% in infectious vs. $2.4 \%$ in non-infectious diarrhea). There were modest differences in race, income, and hospital characteristics across the infectious and non-infectious diarrhea categories.

\section{Correlates of AKI}

The incidence of AKI in infectious and non-infectious diarrheal illness rose with age (Fig. 1). Correlates of AKI by diarrhea type are shown in Table 2; regression models had concordance ("c") statistics of $0.73,0.79$, and 0.79 (corresponding to the area under the receiver operating characteristic curve) for infectious, non-infectious and all diarrheal illness respectively. While women were more likely to be hospitalized with diarrhea than were men, women were significantly less likely to develop diarrheal illness-associated AKI (OR $0.70,95 \%$ CI 0.68 to 0.73 ). There were higher risks of AKI in diarrheal illness among hospitals of larger bed size, and in urban teaching hospitals. Patients with Medicare and Medicaid insurance were more likely to develop AKI than patients with private insurance.

The most important comorbid conditions associated with AKI in hospitalized diarrheal illness are shown in Figure 2. Irrespective of diarrhea type, a diagnosis of CKD was strongly associated with development of AKI. Hypertension was also associated with higher odds of AKI in both diarrhea types. When examining diarrhea type (infectious versus non-infectious) as a predictor of AKI in all diarrheal illness, AKI was nearly two-fold more common among patients with infectious diarrhea (OR 1.90, 95\% CI 1.83 to

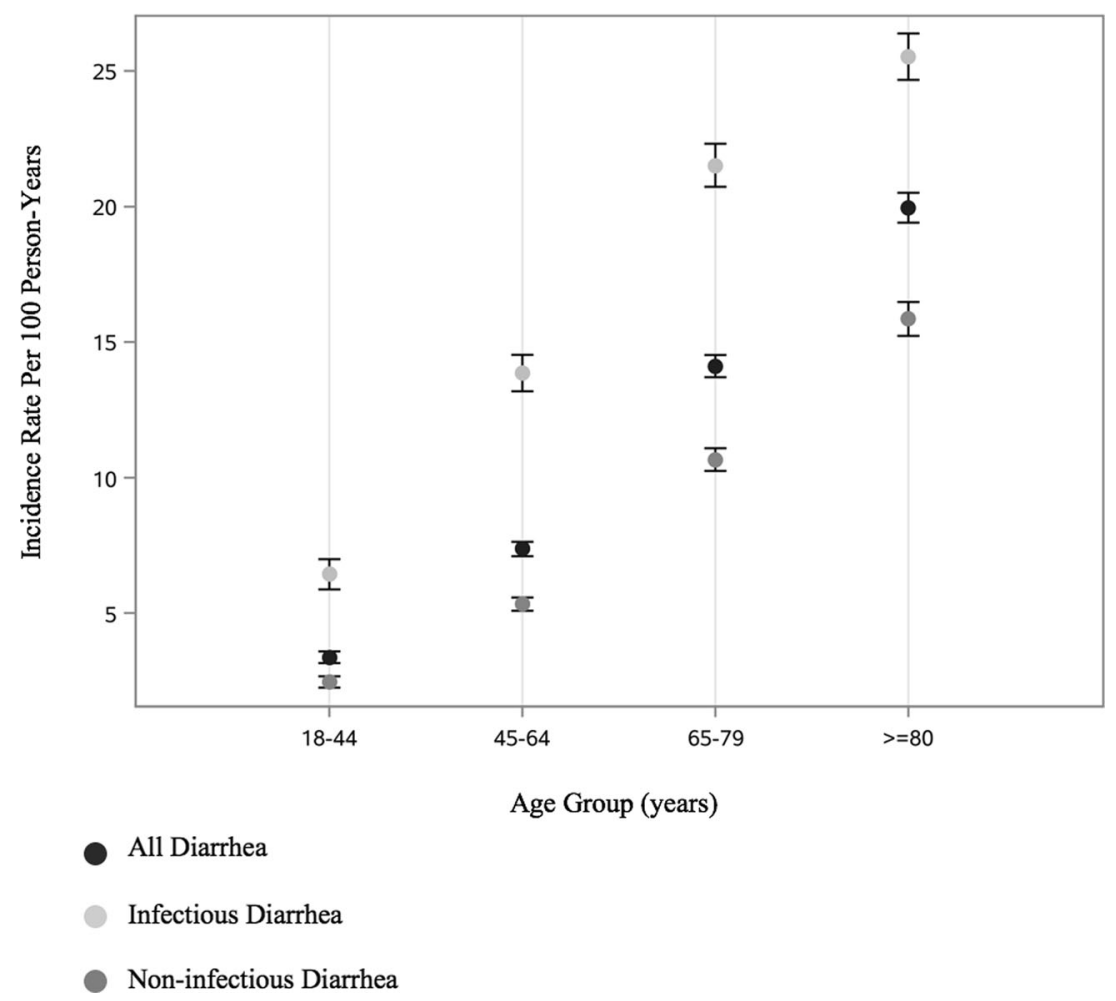

Figure 1 Incidence of AKI in infectious, non-infectious, and all diarrheal illness among hospitalized adults, by age-group (18-44, 45-64, 65-79, and 80 and above). Incidence rates are expressed per 100 person-years. Brackets represent $95 \% \mathrm{CI}$. 
Table 2 Correlates of AKI by Diarrhea Type

\begin{tabular}{|c|c|c|c|}
\hline & $\begin{array}{l}\text { Infectious } \\
\text { diarrhea } \\
(\mathrm{OR}, 95 \% \text { CI })\end{array}$ & $\begin{array}{l}\text { Non-infectious } \\
\text { diarrhea } \\
(\mathrm{OR}, 95 \% \text { CI })\end{array}$ & $\begin{array}{l}\text { All diarrhea } \\
(\mathrm{OR}, 95 \% \mathrm{CI})\end{array}$ \\
\hline \multicolumn{4}{|l|}{ Demographics } \\
\hline Age (per year) & $1.024(1.022-1.026)$ & $1.027(1.025-1.030)$ & $1.027(1.025-1.029)$ \\
\hline Female sex & $0.68(0.64-0.71)$ & $0.71(0.67-0.75)$ & $0.70(0.68-0.73)$ \\
\hline \multicolumn{4}{|l|}{ Race } \\
\hline White & 1.0 & 1.0 & 1.0 \\
\hline Black & $1.03(0.94-1.13)$ & $1.10(1.01-1.20)$ & $1.06(0.99-1.14)$ \\
\hline Hispanic & $0.84(0.75-0.94)$ & $0.74(0.64-0.83)$ & $0.78(0.72-0.84)$ \\
\hline Other* & $0.80(0.69-0.93)$ & $0.93(0.81-1.05)$ & $0.86(0.72-0.84)$ \\
\hline \multicolumn{4}{|l|}{ Hospital details } \\
\hline Elective admission & $0.77(0.65-0.90)$ & $0.60(0.55-0.67)$ & $0.63(0.58-0.68)$ \\
\hline \multicolumn{4}{|l|}{ Teaching status } \\
\hline Urban, teaching & 1.0 & 1.0 & 1.0 \\
\hline Rural & $0.79(0.71-0.87)$ & $0.69(0.62-0.76)$ & $0.74(0.68-0.80)$ \\
\hline Urban, non-teaching & $0.89(0.83-0.96)$ & $0.87(0.81-0.93)$ & $0.88(0.83-0.93)$ \\
\hline \multicolumn{4}{|l|}{ Bedsize } \\
\hline Large & $\mathrm{N} / \mathrm{A}$ & 1.0 & 1.0 \\
\hline Medium & $\mathrm{N} / \mathrm{A}$ & $0.93(0.87-1.00)$ & $0.93(0.88-0.99)$ \\
\hline Small & $\mathrm{N} / \mathrm{A}$ & $0.86(0.79-0.94)$ & $0.89(0.83-0.95)$ \\
\hline \multicolumn{4}{|l|}{ Region } \\
\hline West & 1.0 & 1.0 & 1.0 \\
\hline Northeast & $0.85(0.77-0.94)$ & $0.84(0.76-0.93)$ & $0.85(0.79-0.93)$ \\
\hline Midwest & $0.87(0.78-0.96)$ & $0.95(0.86-1.06)$ & $0.91(0.84-0.99)$ \\
\hline South & $1.00(0.91-1.10)$ & $1.03(0.94-1.13)$ & $1.02(0.95-1.10)$ \\
\hline \multicolumn{4}{|l|}{ Primary payer } \\
\hline Private & $\mathrm{N} / \mathrm{A}$ & 1.0 & 1.0 \\
\hline Medicare & $\mathrm{N} / \mathrm{A}$ & $1.11(1.03-1.20)$ & $1.06(1.00-1.13)$ \\
\hline Medicaid & $\mathrm{N} / \mathrm{A}$ & $1.31(1.17-1.47)$ & $1.20(1.10-1.31)$ \\
\hline Other $\dagger$ & $\mathrm{N} / \mathrm{A}$ & $1.36(1.22-1.51)$ & $1.23(1.17-1.39)$ \\
\hline \multicolumn{4}{|l|}{ Chronic conditions } \\
\hline Diarrhea type, infectious vs non-infectious & N/A & N/A & $1.90(1.83-1.97)$ \\
\hline CKD & $4.05(3.73-4.41)$ & $5.82(5.34-6.33)$ & $4.81(4.52-5.12)$ \\
\hline HTN & $1.31(1.22-1.41)$ & $1.35(1.26-1.44)$ & $1.33(1.27-1.40)$ \\
\hline Cardiovascular disorders & N/A & $1.36(1.29-1.44)$ & $1.21(1.16-1.27)$ \\
\hline Overweight & N/A & $1.16(1.07-1.26)$ & $1.14(1.08-1.22)$ \\
\hline Diabetes & $1.17(1.10-1.25)$ & $1.19(1.12-1.27)$ & $1.17(1.12-1.23)$ \\
\hline Other endocrine & N/A & $1.61(1.35-1.92)$ & $1.35(1.18-1.54)$ \\
\hline IBD & $\mathrm{N} / \mathrm{A}$ & $1.23(1.13-1.33)$ & $1.17(1.10-1.25)$ \\
\hline Other GI & $\mathrm{N} / \mathrm{A}$ & $1.15(1.06-1.25)$ & $1.14(1.10-1.21)$ \\
\hline Solid organ transplant & $1.61(1.31-1.99)$ & $2.13(1.64-2.75)$ & $1.92(1.62-2.27)$ \\
\hline HIV/AIDS & $1.73(1.34-2.23)$ & N/A & $1.59(1.29-1.95)$ \\
\hline Rheumatologic/immunologic disorders & $1.15(1.06-1.26)$ & $1.18(1.09-1.29)$ & $1.17(1.10-1.24)$ \\
\hline Hematologic, BMT & N/A & $1.36(1.28-1.44)$ & $1.22(1.17-1.28)$ \\
\hline
\end{tabular}

Correlates for each diarrhea category were identified using backward step-wise selection with a p value cutoff of $<0.10$ for inclusion *Combines data for Asian/Pacific Islanders, Native Americans, and others

${ }^{\dagger}$ Includes self-pay, no charge, and other payers

1.97). However, the percentage of persons with dialysisrequiring AKI was not different between the two groups: 2.4 and $2.1 \%$ for non-infectious and infectious diarrhea, respectively.

\section{Mortality, Length of Stay, and Costs}

The development of AKI with a hospitalized diarrheal illness was associated with higher mortality, longer lengths of stay and higher costs of stay as compared to not developing AKI (Table 3). Although intermediate outcomes like the need for dialysis or stay in the intensive care unit (ICU) did not differ by primary diarrhea diagnosis (Table 1), persons with noninfectious diarrhea and AKI experienced modestly higher odds of mortality (OR 5.55, 95\% CI 4.75 to 6.48 for noninfectious vs OR $4.18,95 \%$ CI 3.45 to 5.05 for infectious) and substantially longer lengths of stay and higher costs of stay, when compared to persons with infectious diarrhea and AKI.
Age and diarrhea type modified these associations, with the increased odds of mortality, longer length of stay, and higher costs more pronounced at younger ages and among patients with non-infectious diarrhea ( $p$ value $<0.05$ for three secondary outcomes).

\section{DISCUSSION}

Acute kidney injury (AKI) complicated hospitalized diarrheal illness in approximately 1 in 10 persons overall, and 1 in 6 over the age of 65. Development of AKI was associated with a 5 -fold increase in mortality in persons admitted with diarrheal illness. Underlying CKD and hypertension were associated with the risk of AKI in both infectious and non-infectious diarrheal illness requiring hospitalization, likely related to diminished renal reserve and medications commonly used to manage these conditions. 
a

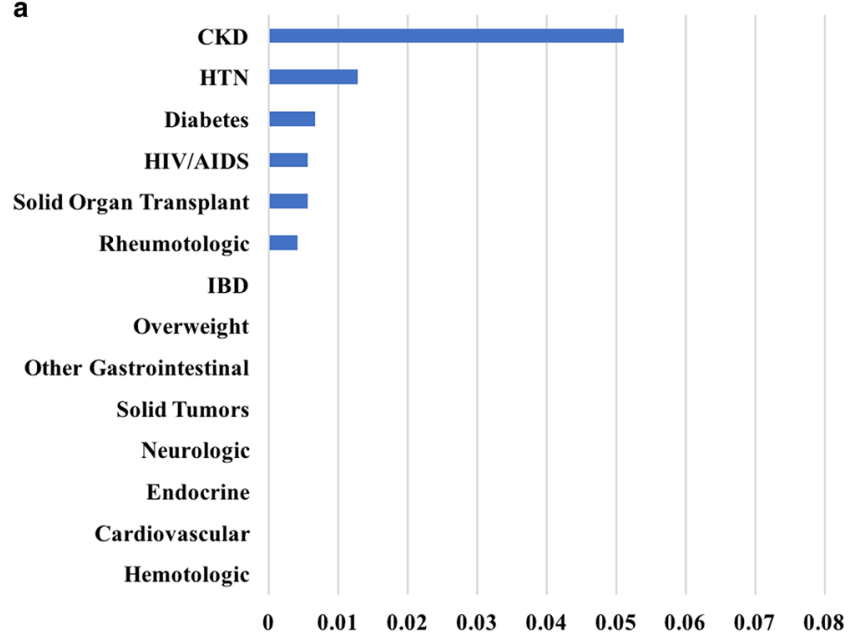

b

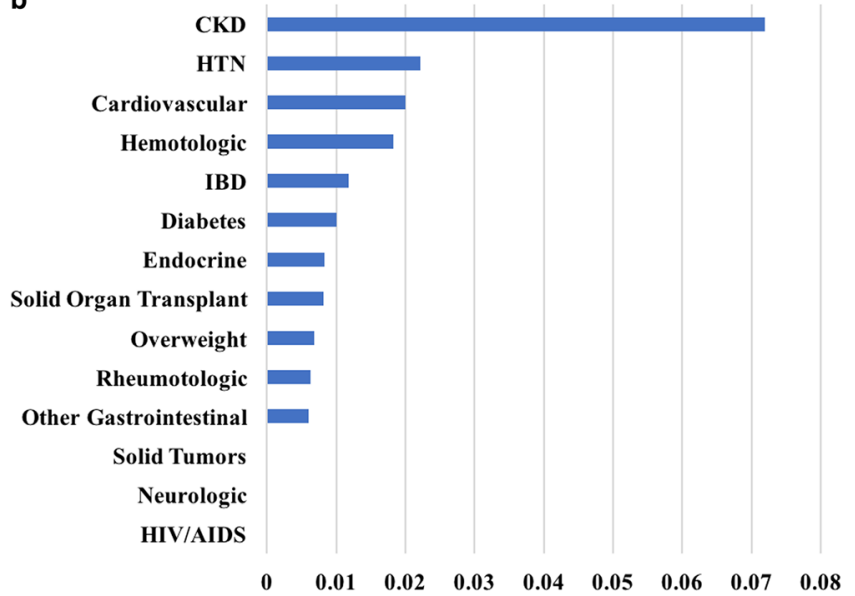

Figure 2 Comorbidities associated with increased odds of AKI in hospitalized infectious diarrheal illness (a). Comorbidities associated with increased odds of AKI in hospitalized non-infectious diarrheal illness (b). Bar length represents relative importance score based on the absolute standardized coefficient.

Our study quantifies risk for AKI in hospitalized diarrheal illness from a large, nationally representative hospital discharge data source. In previously published observational studies that have utilized single-center hospital data, the incidence of AKI in other common, acute causes of hospitalization ranged from $12 \%$ (in urinary tract infections) ${ }^{27}$ to $18 \%$ (in community acquired pneumonia). ${ }^{28}$ In these studies, as in ours, hypertension, $\mathrm{CKD}$, and diabetes were important risk factors for AKI. These findings are in line with the results we observed, particularly if we take into account the under ascertainment inherent in ICD-9 code-based AKI and comorbidity definitions.

Extracellular volume losses from the gastrointestinal tract are often the primary cause of AKI (often described as "prerenal azotemia" in hospitalized diarrheal illness), and it follows that any chronic condition that exacerbates volume disturbance may contribute to renal injury. The findings of our analysis affirm this idea; irrespective of diarrhea type, the presence of CKD and hypertension increased the odds of developing AKI. Chronic kidney disease is a dominant risk factor for AKI in nearly all medical and surgical settings. ${ }^{29-}$

${ }^{31}$ Hypertension may be a manifestation of mild to moderate CKD that may be un- or under-recognized by physicians and other healthcare personnel. Furthermore, medications commonly used for outpatient management of CKD and hypertension could render patients hospitalized with diarrhea more susceptible to AKI. Diuretics impair the kidney's ability to reabsorb sodium and to concentrate the urine, impeding the body's natural physiologic response to volume depletion. Inhibitors of the renin-angiotensinaldosterone system (RAAS), including angiotensinconverting enzyme (ACE) inhibitors, angiotensin receptor blockers (ARBs) and mineralocorticoid receptor antagonists (MRAs), blunt the renal auto-regulatory response at times of hemodynamic disturbance, resulting in a sharp decline in filtration fraction and glomerular filtration rate (GFR) with volume depletion and associated systemic hypotension. ${ }^{32,33}$ Indeed, prior studies have cited use of ACE inhibitors, ARBs and diuretics as risk factors for AKI. ${ }^{27,28}$ The role of antimicrobial medications in causing both AKI and diarrheal illness should also be considered; for example, antibiotic use could lead to both acute interstitial nephritis and Clostridium difficile-associated diarrhea.

Aside from CKD and hypertension, we found that diabetes mellitus, solid organ transplantation, and HIV/AIDS were associated with higher risks of AKI in hospitalized infectious diarrhea, likely due to the immunosuppressive effects of the conditions themselves as well as their treatment (i.e., calcineurin inhibitors for solid organ transplantation). Underlying IBD and cardiovascular disorders increased the risk of AKI in non-infectious diarrheal illness; these conditions are associated with chronic inflammation and vascular compromise that can adversely affect renal function.

Table 3 Effects of AKI in Hospitalized Diarrheal Illness on Death, Length, and Cost of Stay

\begin{tabular}{llll}
\hline \hline & Infectious diarrhea & Non-infectious diarrhea & All diarrhea \\
\hline Death (OR, 95\% CI) $\dagger$ & $4.18(3.45,5.05)$ & $5.55(4.75,6.48)$ & $5.05(4.47,5.72)$ \\
Length of stay, days $\dagger+$ & $1.24(1.10,1.38)$ & $2.07(1.88,2.26)$ & $1.68(1.56,1.80)$ \\
Cost of stay, dollars $\dagger+$ & $2550(2131,2969)$ & $6062(5422,6703)$ & $4411(4023,4800)$ \\
\hline
\end{tabular}

Reference group is patients with hospitalized diarrheal illness who did not develop AKI

${ }^{\prime}$ Separate backward stepwise selection models were run for the three outcomes: death, length, and cost of stay. We adjusted for the Table 1 correlates that were below the $p$ value cutoff of 0.10. All models included adjustments for age, hypertension, diabetes, cardiovascular disorders, pulmonary disorders, underweight status, solid tumors, and hematologic disorders

${ }^{*}$ Mean change versus hospitalizations for diarrheal illness without AKI 
Recognition of comorbid conditions associated with an increased risk of AKI in hospitalized diarrheal illness could aid in early diagnosis and treatment; our analysis also suggests the need for further work investigating prevention. Studies have noted anecdotal benefits of temporary cessation of diuretics and RAAS blockers during acute gastrointestinal illness. ${ }^{34,35}$ In its review of potential measures to prevent AKI, National Institute for Health and Care Excellence (NICE) in the UK recognized diarrheal illness as one of the four most important clinical situations for which temporary cessation of ACE inhibitors or ARB therapy could be considered. After an exhaustive literature review, the Committee did not identify any relevant studies but nonetheless felt that clinicians should consider cessation of diuretics and RAAS blockers during episodes of diarrhea or vomiting. ${ }^{36}$ Others have recommended giving a pocket card to persons at risk for AKI that reminds them to hold these agents during acute illness. ${ }^{37}$ Our data support the need for further evaluation of current practices, and whether temporary discontinuation at the onset of diarrheal illness of medications that affect renal autoregulation could protect against AKI. One particular challenge is judging the severity of the illness versus the benefits of continuation of therapy.

In evaluating the association between AKI and hospitalization outcomes, we found that while AKI was more common in the elderly, younger persons with AKI experienced worse outcomes. Given the better renal reserve and lower comorbidity burden generally observed in this group, it is plausible that development of clinician-recognized AKI in younger persons reflects more severe acute illness, and thereby is associated with higher mortality, and longer lengths of stay and higher costs. ${ }^{38}$ A prior study by Chertow et al. reinforced the construct that the extent of injury required to produce $\mathrm{AKI}$ in persons with baseline normal renal function must be severe; persons with de novo AKI were found to have a higher risk of death when compared to those with AKI on CKD. ${ }^{39}$ Unexpectedly, AKI in non-infectious diarrhea was associated with a more pronounced increase in mortality, length of stay and costs. We hypothesize that delayed diagnosis of AKI may play a role in this outcome. Whereas episodes of infectious diarrhea and its accompanying AKI are often acute in onset, selflimited, and reversible, the insidious nature of many types of non-infectious diarrhea (e.g., malabsorption due to multiple conditions, inflammatory or ischemic bowel disease, or adverse medication effects) may delay physician recognition of AKI.

There are several strengths to our analysis. We utilized the NIS, a highly representative data source capturing a sizeable fraction of all episodes of hospitalized diarrheal illness in the USA. As such, we could adjust for a variety of patient- and facility-specific factors to determine correlates and consequences of AKI complicating this condition. There are also several important limitations. Firstly, NIS data cannot determine the temporal relationship between diarrheal illness and AKI, which only captures information at discharge. We attempted to address this issue by restricting our cohort to persons with a primary, rather than any, diagnosis of diarrheal illness - thus making it less likely that AKI precedes diarrheal illness. Secondly, the lack of laboratory data available in the NIS precludes more sensitive measures of AKI. When using administrative diagnosis codes, there is potential for underreporting and misclassification of diseases. Since AKI captured via administrative codes generally reflects more advanced stages of AKI than alternative means of detection (e.g., with serial laboratory data), data on associated mortality, length of stay, and costs must be taken in context. More granular data might have allowed us to estimate the role medications - particularly diuretics and other antihypertensive agents - might be playing in hospitalized diarrheal illnessassociated AKI. Finally, since NIS captures hospital stays, we cannot estimate the incidence of AKI complicating more mild forms of diarrheal illness that do not require hospitalization. While one could reliably predict that milder cases of infectious diarrhea would be associated with much lower rates of AKI than cases requiring hospitalization, it is possible that persons with indolent, non-infectious diarrhea have a meaningful burden of acute and chronic kidney disease - this topic requires further study.

In summary, we found a high incidence of AKI complicating infectious and non-infectious hospitalized diarrheal illness, particularly among older and elderly individuals, and among persons with underlying CKD, vascular disease, and comorbid conditions that are likely to be immunosuppressive. Given the observed consistent association of CKD and hypertension with diarrheal illness-associated AKI, we recommend proactive management of potential medication and physiologic triggers of AKI in this vulnerable population.

Corresponding Author: Christina Bradshaw, MD; Division of Nephrology, Department of Medicine Stanford University School of Medicine, Stanford, CA, USA (e-mail: cbradsha@stanford.edu).

Funding Information Dr. Bradshaw is supported by the fellowship training grant 5T32DKOO7357 and NIH Fogarty Global Health Equity Scholars TW009338. Dr. Anand is supported by NIDDK K23DK101826-03.

\section{Compliance with Ethical Standards:}

Conflict of Interest: The authors declare that they do not have a conflict of interest.

\section{REFERENCES}

1. World Health Organization. Diarrhoeal disease. 2017, May; Available from: http://www.who.int/mediacentre/factsheets/fs330/en/. Accessed August 2017.

2. UNICEF. Diarrhoeal Disease: Current Status + Progress. 2018, March. Available from: https://data.unicef.org/topic/child-health/diarrhoealdisease/. Accessed July 2017.

3. Evans CT, Safdar N. Current trends in the epidemiology and outcomes of clostridium difficile infection. Clin Infect Dis. 2015;60 (Suppl 2):S66-71. 
4. Lessa FC, et al. Burden of Clostridium difficile infection in the United States. N Engl J Med. 2015;372(9):825-34.

5. Yadav S, et al. A population-based study of incidence, risk factors, clinical spectrum, and outcomes of ischemic colitis. Clin Gastroenterol Hepatol. 2015; 13(4):731-8.e1-6; quiz e41.

6. Teetor $\mathbf{T}$, et al. the changing epidemiology of diverticulitis in the United States. Am Surg. 2017;83(4):134-136.

7. Bharucha $\mathbf{A E}$, et al. temporal trends in the incidence and natural history of diverticulitis: a population-based study. Am J Gastroenterol. 2015;110(11):1589-96.

8. Greenberg JH, Coca S, Parikh CR. Long-term risk of chronic kidney disease and mortality in children after acute kidney injury: a systematic review. BMC Nephrol. 2014; 15:184.

9. Coca SG, Singanamala S, Parikh CR. Chronic kidney disease after acute kidney injury: a systematic review and meta-analysis. Kidney Int. 2012;81(5):442-8.

10. Bucaloiu ID, et al. Increased risk of death and de novo chronic kidney disease following reversible acute kidney injury. Kidney Int. 2012;81(5): 477-85.

11. HCUP NIS Database Documentation. Healthcare Cost and Utilization Project (HCUP). 2018. Agency for Healthcare Research and Quality, Rockville. www.hcup-us.ahrq.gov/db/nation/nis/nisdbdocumentation. jsp. Accessed March 2018.

12. Kilgore PE, et al. Trends of diarrheal disease-associated mortality in US children 1968 through 1991. JAMA. 1995;274(14):1143-8.

13. Collins CE, et al. Epidemiology and outcomes of community-acquired Clostridium difficile infections in Medicare beneficiaries. J Am Coll Surg. 2014;218(6):1141-1147.e1.

14. Wheat CL, Strate LL. Trends in Hospitalization for Diverticulitis and Diverticular Bleeding in the United States From 2000 to 2010. Clin Gastroenterol Hepatol. 2016;14(1):96-103.e1.

15. Lopman BA, et al. Increasing rates of gastroenteritis hospital discharges in US adults and the contribution of norovirus, 1996-2007. Clin Infect Dis. 2011;52(4):466-74.

16. Curns AT, et al. Reduction in acute gastroenteritis hospitalizations among US children after introduction of rotavirus vaccine: analysis of hospital discharge data from 18 US states. J Infect Dis. 2010;201(11): 1617-24

17. Hsu RK, et al. Temporal changes in incidence of dialysis-requiring AKI. J Am Soc Nephrol. 2013;24(1):37-42.

18. Lenihan CR, et al. Trends in acute kidney injury, associated use of dialysis, and mortality after cardiac surgery, 1999 to 2008. Ann Thorac Surg. 2013;95(1):20-8.

19. Waikar SS, et al. Validity of international classification of diseases, ninth revision, clinical modification codes for acute renal failure. J Am Soc Nephrol. 2006; 17(6):1688-94

20. Grams ME, et al. Performance and limitations of administrative data in the identification of AKI. Clin J Am Soc Nephrol. 2014;9(4):682-9.
21. Blecker S, et al. Heart failure-associated hospitalizations in the United States. J Am Coll Cardiol. 2013;61(12):1259-67.

22. Lee CS, et al. Comorbidity profiles and inpatient outcomes during hospitalization for heart failure: an analysis of the U.S. Nationwide inpatient sample. BMC Cardiovasc Disord. 2014;14:73

23. Tsai WS, Inge TH, Burd RS. Bariatric surgery in adolescents: recent national trends in use and in-hospital outcome. Arch Pediatr Adolesc Med. 2007;161(3):217-21.

24. Silver SA, et al. Cost of acute kidney injury in hospitalized patients. J Hosp Med. 2017;12(2):70-76.

25. Blough DK, Madden CW, Hornbrook MC. Modeling risk using generalized linear models. J Health Econ. 1999;18:153-171.

26. Malehi AS, Pourmotahari F, Angali KA. Statistical models for the analysis of skewed healthcare cost data: a simulation study. Health Econ Rev. 2015;5:11.

27. Hsiao CY, et al. Risk factors for development of acute kidney injury in patients with urinary tract infection. PLoS One. 2015;10(7):e0133835.

28. Akram AR, et al. Incidence and prognostic implications of acute kidney injury on admission in patients with community-acquired pneumonia. Chest. 2010;138(4):825-32.

29. Waikar SS, Liu KD, Chertow GM. Diagnosis, epidemiology and outcomes of acute kidney injury. Clin J Am Soc Nephrol. 2008;3(3):844-61.

30. Thakar CV, et al. Influence of renal dysfunction on mortality after cardiac surgery: modifying effect of preoperative renal function. Kidney Int 2005;67(3): 1112-9.

31. Hoste EA, et al. Acute renal failure in patients with sepsis in a surgical ICU: predictive factors, incidence, comorbidity, and outcome. J Am Soc Nephrol. 2003; 14(4):1022-30.

32. Mason NA. Angiotensin-converting enzyme inhibitors and renal function. DICP. 1990;24(5):496-505.

33. Bauer JH. Role of angiotensin converting enzyme inhibitors in essential and renal hypertension. Effects of captopril and enalapril on reninangiotensin-aldosterone, renal function and hemodynamics, salt and water excretion, and body fluid composition. Am J Med. 1984;77(2A):4351.

34. Stirling C, et al. Diarrhoea, vomiting and ACE inhibitors:-an important cause of acute renal failure. J Hum Hypertens. 2003;17(6):419-23.

35. Liu P, Nikels M, Combs B. Diuretics and diarrhea: a dangerous combination: a teachable moment. JAMA Intern Med. 2014;174(2):182.

36. Acute kidney injury: prevention, detection and management up to the point of renal replacement therapy. 2013 August. National Institute for Health and Care Excellence (NICE).

37. Harty J. Prevention and management of acute kidney injury. Ulster Med J. 2014;83(3):149-57.

38. Kaddourah A, et al. Epidemiology of acute kidney injury in critically ill children and young adults. N Engl J Med. 2017;376(1):11-20.

39. Chertow GM, et al. Acute kidney injury, mortality, length of stay, and costs in hospitalized patients. J Am Soc Nephrol. 2005; 16(11):3365-70. 